\section{5 \\ SingiLogos}

SINGILOGOS 2021, 1(1): 152 - 167

ISSN: $2812-7005$

UDK: $27-183.7$

$27-423.4$

$274 / 278-423.4$

DOI: 10.18485/us_singilogos.2021.1.1.11

Original paper/Originalni naučni rad

\title{
LE PÉCHÉ, LA LIBERTÉ ET LE LIBRE ARBITRE
}

\author{
Stavros Yangazoglou* \\ Professeur assistant de théologie dogmatique à l'Université, \\ Nationale et Capodistrienne d'Athènes
}

\section{Article info:}

Received: April 27, 2021

Correction: June 23, 2021

Accepted: July 27, 2021 quel est le sens existentiel de la vie et comment une discussion sur l'anthropologie pourrait contribuer au monde, à I'homme et à l'histoire, du point de vue de la théologie orthodoxe et aussi de l'enseignement de Luther, doit être explorée. Dans le nouveau cadre du pluralisme, la théologie chrétienne est appelée à articuler une proposition et à donner un sens à la vie non seulement pour le monde, mais pour I'homme et pour I'histoire. Dans cette perspective, il doit formuler une nouvelle approche herméneutique de l'anthropologie chrétienne, en distinguant ce qui appartient aux fondamentaux, et ce qui est en ce sens immuable, de ce qui est simplement l'ordre du langage culturel ou de ce qui est historiquement lié au temps. Dans cette herméneutique théologique, face au pluralisme et à l'altérité dans sa dimension œcuménique, la théologie chrétienne doit retrouver le sens dynamique de l'existence humaine en dialogue avec les angoisses et les problèmes de I'homme d'aujourd'hui. Malgré la relation profonde entre la modernité et l'évolution de la tradition chrétienne en Europe, il est temps de souligner que les Églises chrétiennes ne sont plus la puissance active de l'histoire mais sont en revanche, le spectateur passif de bouleversements et événements. Les réformes sociales et économiques, la libération et l'émancipation des peuples et des classes sociales, les effets désastreux du nationalisme et du totalitarisme, le progrès technologique, le nihilisme consumériste et la destruction catastrophique de l'environnement naturel, le nouvel ordre des choses, les formes postmodernes de la «spiritualité» ainsi que toute tentative d'échapper aux difficultés de la vie, etc. sont l'œuvre de facteurs ancrés dans le monde actuel, coupés de la foi chrétienne, même en opposition avec elle. Si Luther vivait à notre époque, ses priorités théologiques seraient sans aucun doute très différentes. Il pourrait être une initiative alternative pour la réconciliation des chrétiens divisés et un témoignage public concret du salut du Christ dans le monde d'aujourd'hui, dans lequel, au-delà du péché individuel, règne le mal collectif et généralisé sous toutes ses formes.

\section{Mots-clés:}

Tradition patristique, Réforme,

Luther, anthropologie, herméneutique, création, image et ressemblance, libre arbitre, serf arbitre, péché, liberté, divinisation, synergie, salut, modernité, rédemption, justification. 
Cinq siècles après la Réforme de Luther, dans un contexte de modernité avancée, tous les grands récits et les traditions étant fatalement relégués à la marge, quels peuvent bien être l'opportunité et le sens existentiel de la vie pour le monde, I'homme et l'histoire, d'un questionnement de nature anthropologique du point de vue de la théologie orthodoxe mais également de l'enseignement de Luther? Dans le cadre nouveau du pluralisme, la théologie chrétienne est appelée à articuler une proposition et à donner un sens à la vie pour le monde, pour l'homme et pour l'histoire. Dans cette perspective, elle doit formuler une nouvelle approche herméneutique de l'anthropologie chrétienne, en distinguant ce qui appartient aux fondements, et qui est en ce sens immuable, et ce qui relève simplement de l'ordre du langage culturel et mondain ou ce qui est historiquement lié à l'époque. Dans cette herméneutique théologique, face au pluralisme et à l'altérité dans sa dimension œcuménique, la théologie chrétienne se doit de retrouver le sens dynamique de l'existence humaine en dialogue avec les angoisses et les problèmes de I'homme d'aujourd'hui.

\section{L'HOMME ET LE MONDE}

La création doit son existence à la libre volonté et à l'amour de Dieu. En tant qu'existence créée, elle a été engendrée du non-être et n'est pas éternelle. Elle aurait pu également ne pas être. Son existence est un don et une grâce. ${ }^{1}$ Dieu crée l'homme lorsque le cycle de la création à partir de rien des êtres intelligents et sensibles est à son terme, comme un achèvement et un couronnement. Seul I'homme, de tous les êtres intelligents possède à la fois un corps et une âme. L'homme, en tant que composé d'un corps et d'une âme est un produit de la création, il en est le couronnement et une expression personnelle. Ce maximalisme anthropologique de la tradition biblique et patristique se rapporte précisément au fait qu'il est composé d'un corps et d'une âme. ${ }^{2}$ La création est un don que Dieu fait à l'homme et celui-ci s'y installe comme un roi. En même temps, I'homme est à lui seul un microcosme. L'homme et le cosmos sont profondément imbriqués l'un dans l'autre. L'homme sans le monde et le monde sans l'homme n'ont aucune raison d'exister. En tant qu'image du monde et image de Dieu, I'homme a la responsabilité et la mission d'exercer son libre arbitre de sorte qu'il puisse, le moment venu, communier pleinement avec Dieu et entraîner ainsi le reste de la création vers la vie divine. C'est en cela précisément que réside l'objectif de la création de l'homme. Il s'agit là de la mission hiératique de l'homme, qui a un rôle à jouer en venant compléter celle du roi. La création du monde et de l'homme n'est pas un événement accompli, mais il était et continue à être orienté dynamiquement vers une plénitude eschatologique, le sens du monde et de l'homme se trouvant en Dieu lui-même. Sans communion et sans relation avec Dieu, toute existence créée est sans cesse menacée de retourner au non-être. Cette conception montre parfaitement que la création de l'homme implique dès le départ des présupposés christologiques. Le Verbe créateur de Dieu devait d'emblée s'unir à l'homme et non à un quelconque élément de la création, car l'homme est le seul être qui constitue un lien et une articulation

1 Zizioulas, 1984,153-173.

2 Cf. Psaumes 8, 5: «A peine le fis-tu moindre qu'un dieu; tu le couronnes de gloire et de beauté ». Irénée de Lyon, Contra haereses V, 6, 1, PG 7, 1137A. Grégoire Palamas, The One Hundred and Fifty Chapters, 61,154 . 
entre tous les êtres créés. Le premier homme créé est une "personnalité corporative », l'ancêtre du genre humain. L'homme a été créé à l'image et à la ressemblance de Dieu car, d'emblée, son objectif était d'établir une communion constante et continue avec la vie de Dieu. ${ }^{3}$ Dans l'aventure dramatique et le parcours de la création et de l'homme, Dieu lui-même va intervenir selon un mode désormais paradoxal. Le Verbe divin a créé I'homme à son image et à sa ressemblance afin de s'unir à lui.

\section{IMAGE ET RESSEMBLANCE}

L'homme, en tant qu'existence constituée d'un corps et d'une âme est l'image unique et exclusive dans la création du Dieu trinitaire. Au-delà de la physiologie du corps physique et de l'esprit immatériel, I'homme a une relation particulière et un rapport existentiel avec le Dieu créateur, car il reçoit la grâce (ou l'énergie) de l'Esprit Saint. Cette composante pneumatologique de l'homme renvoie en premier lieu au Dieu trinitaire, puisqu'elle lui est accordée par le Verbe du Père. L'homme représente le Verbe divin, et son existence repose sur la grâce du Saint-Esprit. ${ }^{4}$ Sans ce souffle vivifiant, l'homme ne serait qu'un simple élément anonyme et impersonnel de la création, sans relation particulière et sans perspective aucune de s'unir et de ressembler au Créateur. Cette voie vers la ressemblance avec Dieu ne peut en aucun cas être donnée d'office, dans le cadre d'une évolution naturelle, comme une adaptation éthique ou une disposition réglementaire, mais elle ne peut être au contraire que progressive à travers la liberté de l'homme doté de libre arbitre. ${ }^{5}$

Selon la théologie des Pères grecs de l'Église, l'image de Dieu en I'homme se fait jour principalement dans le fait qu'il est doté par nature de libre arbitre ${ }^{6}$ et qu'il est en mesure d'exister en tant que personne libre. Autrement dit, l'homme désire exister parce qu'il est animé par l'amour, répondant au mouvement qui porte Dieu à l'aimer. L'entendement, qui est en lien avec le verbe et l'élan spirituel de l'homme, exprime cette idée que l'image est liberté et épectase de l'existant créé.7 Il ne s'agit rien de moins que l'antique valeur de la liberté humaine, dont l'exercice, en relation directe avec l'Esprit Saint qui lui est inhérent, ne viserait pas simplement à aligner sa vie sur celle du Dieu trinitaire mais fonderait sa vie sur la sienne. L'homme ne pense pas ou ne se comprend pas comme étant à l'image de Dieu, mais il a la possibilité de communier et de rester en rapport empiriquement et existentiellement avec lui, autrement dit dans un rapport interpersonnel, en faisant l'exercice de sa liberté. Participant à l'existence de l'ensemble des êtres créés et communiquant avec Dieu auteur de la création, I'homme a pour mission d'arborer et de mettre en lumière l'ensemble de la création divine, remplissant précisément son rôle d'image de Dieu. Cette conception marque la perspective christologique de I'image de Dieu en I'homme. ${ }^{8}$

3 Grégoire de Nazianze, Oratio 45, In sanctum pascha 7, PG 36, 632AB.

4 Cf. Yangazoglou, 2001, 66-77.

5 Grégoire de Nysse, In Faciamus hominem, PG 44, 273A. Basile de Césarée, De hominis structura 20, PG 30, 29 D.

6 Maxime le Confesseur, Scholia, PG 4, 308A.

7 La signification paulinienne de l'épéctasis comme perfection dans le Christ (Phil. 3,13) est répétée par Origène, Basile de Césarée et est développée principalement par Grégoire de Nysse (De vita Mosis, Homélies sur le Cantique des Cantiques) Cf. Daniélou, 1944, 291-307.

8 Cf. Col. 1,15-18. Maxime le Confesseur, Quaestiones ad Thalassium 60, PG 90, 621A. Grégoire Palamas, Homélies 60, 20. Nicolas Cabasilas, La vie en Christ 2, 65-66. 6, 58. Cf. Zizioulas, 1984. 


\section{LIBERTÉ ET MUTABILITÉ}

L'homme, bien qu'il soit l'image la plus proche de Dieu, n'en est pas moins pour autant un être créé, un être engendré à partir de rien. La mutabilité est inhérente à sa nature créée, à l'opposé de celle de Dieu, comme sa principale composante. Aucune « entéléchie » naturelle ne surpasse l'homme en tant qu'être créé. Aucune « grâce de la création », donnée dans sa nature, ne peut conjurer sa mutabilité et donc, par conséquent, son éventuel retour au non-être. Ayant été créé à partir du néant, le non-être est toujours présent en tant que menace existentielle. Pour que l'être créé de I'homme puisse s'affranchir des conséquences néfastes d'une telle éventuelle menace, il est nécessaire qu'il s'implante librement dans une réalité ontologique au-delà d'elle et extérieure à elle, dans une existence non créée, qui n'est pas susceptible de mutabilité et qui ne risque pas de sombrer dans le non-être. Le « commencement » créateur de toute existence créée, la vie non créée de Dieu, est l'ancrage unique au-delà de toute existence créée. Dans cette perspective, la création de l'homme à l'image et à la ressemblance de Dieu n'a pas de sens hors du libre arbitre et du caractère volontaire de la logique de la nature. Le progrès des « protoplastes » vers la ressemblance avec Dieu était une possibilité, qui supposait la totale et libre synergie de l'homme à l'appel de Dieu. Librement, I'homme était invité à répondre à cet appel et à entrer en communion avec lui. La mutabilité innée de la nature humaine est portée, selon le libre arbitre, soit vers l'être soit vers le non-être. L'exercice positif du libre arbitre projette et conduit I'homme vers la vie de Dieu. Dans ce but, parallèlement au libre arbitre, Dieu fit don à l'homme d'autres données à son image, de sorte que l'élan de vie de l'homme soit porté dans sa complétude. Le maintien et l'accomplissement de sa relation avec Dieu est une possibilité, et en même temps toutefois une épreuve et un défi pour un être doté de libre arbitre.

\section{LE PÉCHÉ COMME DÉGRADATION DE LA VIE}

Pendant un temps, les « protoplastes » mettaient librement et avec éclat leur suggestion-injonction au service d'un progrès vers la ressemblance avec Dieu. Les derniers arrivés et les tout premiers, toutefois, avec une connaissance et une expérience encore réduites et conventionnelles de la vertu, ont imaginé, imbus d'orgueil, qu'avec exclusivement leurs qualités propres, sans le soutien de Dieu, ils parviendraient à gagner l'immortalité. Leur esprit vaniteux est dominé par la tentation d'égaler Dieu, non à travers une relation soutenue avec Dieu, à travers un mouvement persévérant vers Dieu, mais par une voie autonome et indépendante. S'ils parviennent à goûter au fruit défendu, ils participeront à l'essence de Dieu. À l'homme est proposée l'infidélité démoniaque au plan de Dieu, le dépassement de ses limites d'être créé, sa divinisation et son conflit avec Dieu. Parallèlement, I'homme décide de devenir lui-même le centre de référence ultime de la création dans son ensemble. Ainsi il cède à la «tentation démoniaque », à « I'illusoire divinisation $»^{9}$ et se révolte librement contre Dieu. La possibilité originelle qu'a

9 Cf. Jean Damascène, In Dormitionem Mariae 2, PG 96, 725A. 
I'homme de ressembler à Dieu, de sorte qu'il pourrait entraîner l'union de l'ensemble de la création vers la vie de l'être non créé est brutalement arrêtée. C'est librement qu'il a rejeté et qu'il a abandonné l'élan de la grâce, qui était sa référence ultime et le noyau de son existence, en mettant un terme au plan divin. Il ne s'agit pas là simplement d'un comportement négatif sur le plan éthique, lié à la nature psychique de l'homme, mais avant tout d'un choix à caractère ontologique. L'homme réalise sa vie de façon autarcique et autonome, renonçant à son rapport avec Dieu, à toute communion avec lui. La racine du péché réside dans le fait que l'homme devient « sa propre idole », un dieu à la place de Dieu. Ainsi il s'en remet simplement à ses forces propres, aux facultés de sa nature d'être créé.

L'éloignement de la grâce de l'Esprit Saint a pour conséquence l'état de péché. Les ténèbres et les brumes recouvrent l'esprit de I'homme doté de libre arbitre, qui contient dans son essence le péché occulté derrière des notions, des discours et des actions corrompues. Le plaisir et la douleur du péché paradoxalement lui procure une jouissance dans la dépravation et le malheur. L'esprit fait naître dans le corps l'amour de la matière, y introduit la vie mortelle, une forme de vie aliénée pour l'homme détaché de la vie de Dieu. Ainsi les conséquences de la dégradation, par rapport à la vraie vie, se propagent à l'homme tout entier en tant qu'unité d'un esprit et d'un corps. Dans un premier temps, c'est l'esprit qui est aliéné avant que ne vienne le tour du corps. Lorsque l'esprit se sépare du corps, au moment de la mort, le corps se désagrège. Mais l'âme elle-même, sans la grâce du Saint-Esprit, se nécrose, car l'immortalité n'est pas une propriété naturelle de l'âme, mais bel et bien un don intentionnel de la grâce. Si l'âme est conservée après la chute, ne serait-ce que dans un état nécrosé, ce n'est pas parce qu'elle immortelle dans son essence, mais parce qu'elle est liée à une perspective de réhabilitation dans le Christ de l'homme dans son entier. La dérive vers la négation de la mort correspond au dernier soubresaut de l'existence humaine. De toute évidence, Dieu ne punit pas l'homme selon la loi, ne serait-ce que parce qu'il a exercé à mal sa liberté, mais il subit les conséquences existentielles de sa liberté. Dieu, selon la théologie patristique, n'intervient pas pour abolir de force la décision libre des premiers hommes. Se fait jour ainsi le fond du mystère de la liberté que Dieu a accordée aux hommes.

Mais Dieu n'a pas permis à la mort d'anéantir l'homme, qui même s'il a renoncé à ressembler à Dieu, n'a pourtant pas totalement perdu l'image de Dieu qu'il porte en lui. Comptant, comme à l'origine, sur le repentir futur de l'homme, sur sa ressaisie et sa synergie, Dieu intervient avec philanthropie, non dans le libre arbitre de I'homme, mais dans l'immédiateté des conséquences de l'exercice négatif de ce libre arbitre. La désobéissance et la révolte n'a pas entraîné directement et aussitôt la mort, car demeurait l'espoir que l'homme se ressaisisse par l'exercice de son libre arbitre. Le fossé provoqué par le péché originel dans la relation entre le créé et l'incréé peut être corrigé. Le plan de Dieu n'a pas changé. Le genre humain était du reste mortel avant la mort en tant qu'il était de l'ordre du créé. L'économie adamique sera suivie d'une nouvelle perspective. Le péché et l'introduction de la mort n'a pas déjoué le plan de Dieu, mais l'a simplement modifié en fonction du nouvel état engendré par la chute et la mort. Ainsi la perspective christologique primitive prend désormais une forme nouvelle. 
L'image de Dieu chez l'homme est entachée, mais elle n'est pas éteinte. ${ }^{10}$ Son libre arbitre, quoiqu'affaibli, est toujours présent et garde la possibilité, en prenant conscience d'avoir manqué son but, de se tourner de nouveau vers Dieu. ${ }^{11}$ L'extinction totale et définitive est momentanément éloignée dans un souci philanthropique.

Une nouvelle ère commence de sorte que l'homme, progressivement formé, puisse volontairement renouer sa relation d'adoption divine et faire progresser dans sa plénitude sa relation avec Dieu. C'est dans ce but que Dieu accorde aux hommes « les tuniques de peaux » (Genèse 3,21), c'est-à-dire une vie provisoire et corruptible, un ternissement en l'homme de l'image de Dieu mais en même temps un rappel constant de son abandon de la vraie vie et de son sens. Toute une culture de l'homme s'élabore à la suite de l'expérience du péché et de la mort. Le mariage, le travail, la technologie, la science, l'art, la politique, autrement dit toutes les activités et les engagements de la vie d'après la chute, constituent les nouvelles possibilités de survie de l'homme, qui sont des dons et des grâces de Dieu accordés dans le but qu'elles soient développées et qu'elles servent à leur tour le plan de salut par le Christ. Les tuniques de peau ${ }^{12}$ pourront se métamorphoser, le moment venu, en chair revêtue des tuniques du Christ. Au contraire, l'autonomisation ultérieure des activités de la vie accentue l'enfermement de l'homme dans la vie nécrosée, renforce les embûches du péché. Le genre humain survit, voué à la mort ; la vie devient synonyme de la mort, de sorte que le mal du péché ne devienne pas immortel. Il s'agit là d'une pédagogie de la mort. L'homme des tuniques de peau, enfermé dans la conviction de la chair, lutte contre la mortalité à travers sa vie biologique. Cette mort est la mort et la dégradation du corps mortel des tuniques de peau, elle n'est pas la mort de l'homme créé à l'image de Dieu. Par l'exercice du libre arbitre, I'homme des tuniques de peau est appelé à renverser la condition des «protoplastes ».

La relation manquée des premiers hommes à la vie de Dieu et leur aliénation sont transmises comme un mal de la nature humaine à leurs descendants, qui à leur tour, partagent la même nature corruptible des tuniques de peau..$^{13} \mathrm{Ils}$ n'héritent pas toutefois de leur culpabilité personnelle et de leur échec. ${ }^{14}$ Pourtant, en tant que descendants d'Adam et Ève, nous sommes plus condamnables et plus coupables qu'eux, car nous demeurons dans le péché, alors que nous n'ignorons rien de ses conséquences mortelles. De cette façon, la responsabilité personnelle et la culpabilité des premiers hommes devient notre propre responsabilité, notre péché hérité des premiers hommes mais non inéluctable et transmis automatiquement, comme l'a enseigné Augustin, suivi par Luther. Quoi qu'il en soit, le péché originel ne s'identifie pas à la sexualité humaine (concupiscentia). Le péché dans la chair (Romains 8,3) concerne I'homme primitif livré à la corruption et à la mort, qui vit coupé de Dieu. Pourtant, la corruption et la mort comme mal naturel sont transmises à travers la chaîne biologique.

10 Basile de Césarée, De hominis structura 20, ibid. Grégoire Palamas, The One Hundred and Fifty Chapters, 39,126.

11 Nissiotis, 1981.

12 Cf. Nellas, 1987.

13 Grégoire de Nazianze, Oratio 45, In sanctum pascha 7, PG 36, 633A. Pour « les tuniques de peau », Cf. Nellas, 1987, 51.

14 Cf. Cyrille d'Alexandrie, Explanatio in Epist. ad Rom. 5,18, PG 74, 788-789. Jean Chrysostome, In Epist. ad Ephes. 4,1, PG 62, 31. In Epist. ad Rom. 102, PG 60, 477 
Le péché, toutefois, qu'il soit de la nature ou de l'individu, est un état morbide, une ontologie de la corruption pour le genre humain, qui est désormais enfermé dans une vie corrompue. Toutefois, la perspective christologique, commence à se faire entendre dans le récit de l'Ancien Testament, où dans I'histoire traversée de conflits et de contradictions du peuple de Dieu, se fait jour déjà l'espoir du salut et du rachat du genre humain. Malgré les nombreux justes et proches de Dieu, qui par des actions responsables et libres participèrent au plan du salut, aucun n'était sans péché de sorte qu'il soit jugé en fonction de la faute traumatique originelle, du cercle vicieux de la vie et de la mort. L'Ancien Testament raconte, soit symboliquement et métaphoriquement, soit historiquement et prophétiquement, la préparation progressive de cette économie du salut (Heilsgeschichte), qui se réalisera avec l'incarnation de Dieu dans le Christ comme recréation dans la nature et l'histoire de la création.

\section{INCARNATION ET DIVINISATION}

L'homme, seul être du monde créé doté ou non de raison avait le privilège d'opérer I'union du créé et de l'incréé. L'image de Dieu et le libre arbitre de l'homme continuent à exister après la chute et le péché. Par conséquent, c'est à travers l'homme que devait de nouveau se reconstituer et se redresser la création. Pour que le monde puisse dépasser la corruptibilité inhérente à sa nature en tant qu'elle a été créée, corruptibilité qui constitue une menace permanente après la chute, il était nécessaire que l'incréé intervienne avec le libre consentement du créé. Le problème de la dégradation et de la mort n'étant pas un problème d'ordre éthique ou juridique -car il existait dès le départ, en tant que possibilité, du fait de la nature créée et finie des êtres de la création- le salut des êtres créés constituait le problème existentiel par excellence. Cette initiative du Dieu incréé ne devait pas simplement signifier une subordination et un pardon de caractère éthique ou une intervention extérieure nécessaire. Donc, l'homme seul, pas plus que l'ange seul, pas plus que Dieu seul, sans la libre participation et le consentement de I'homme, n'étaient en mesure de ramener la nature créée dans la vie de l'incréé. Cette conception de la pensée patristique rend la volonté éternelle de Dieu, « le mystère éternel et inconnu des anges » de l'incarnation du verbe. ${ }^{15}$

C'est Dieu qui détient la solution du drame et qui peut faire sortir de l'impasse l'existence des êtres créés condamnés à la corruption et à la mort. Son intervention dans la tragédie humaine se réalise en toute liberté, sans entamer ou anéantir la liberté et l'intégrité de l'homme. La sortie de la christologie de l'impasse de l'économie d'Adam devrait signifier un dépassement de la mort, non en tant que principe extérieur et transcendant, mais à l'intérieur même de la nature du créé qui l'assume volontairement. Le dépassement de la corruption était nécessaire pour embrasser I'humanité tout entière et non un individu particulier. Un nouvel Adam était nécessaire qui disposerait d'une hypostase qui lui permettrait de constituer le support personnel

15 Cf. Col. 1, 26-27: « ce mystère resté caché depuis les siècles et les générations et qui maintenant vient d'être manifesté à ses saints : Dieu a bien voulu leur faire connaître de quelle gloire est riche ce mystère chez les païens : c'est le Christ parmi vous ! l'espérance de la gloire ! ». Cf. Col. 2, 2. 4, 3. Rom. 16, 25. Eph. 3, 4. 5, 32. 
de l'intervention divine, d'établir un rapport avec Dieu et d'intégrer en quelque sorte dans son existence l'ensemble des personnes humaines, dépassant ainsi les diverses scissions au sein de l'existence créée. Par ailleurs, l'existence humaine, sur laquelle se réaliserait l'intervention salvatrice devait être accomplie dans son entier, selon le corps et selon l'esprit. Le dépassement de la mort devrait en effet être assumé par ceux qui sont déjà morts. L'existence humaine qui accepterait la présence du Dieu incréé devrait être libérée du péché. Quoi qu'il en soit, une telle énergie salvatrice à l'intérieur même de la nature et du fonctionnement de l'humanité, ne pourrait être celle d'un ange ou d'un homme quelconque, mais uniquement celle de Dieu incarné. Dans le cadre de la création de l'homme à l'image et à la ressemblance de Dieu, la tradition patristique dans son ensemble souligne avec force la relation archétypale du Verbe de Dieu avec l'existence de l'homme. ${ }^{16}$ L'intervention paradoxale du Fils était incluse d'une certaine façon dans l'évolution positive de l'économie d'Adam en tant qu'union progressive du créé et de l'incréé. Le Christ n'est le résultat ni du péché ni du diable. Si Augustin ainsi que Luther recentrent leur enseignement sur le péché originel -si homo non periisset, Filius hominis non venisset ${ }^{17}$ - les Pères grecs insistent sur l'incarnation comme exécution du plan originel de la création de I'homme: "Il s'est fait homme pour que nous devenions dieux ${ }^{18}$. Cette christologie «première », sans changer d'orientation, opère à présent un déplacement, pour tenir compte de l'événement tragique de la chute et de la corruption du genre humain. L'incarnation était l'objectif initial et absolu de Dieu dans l'acte de la création. Le genre humain « mortel et destiné à la mort de par sa nature ${ }^{19}$ avait besoin d'un couronnement, d'un salut et d'une union avec l'Archétype. L'événement christologique n'a pas pour cause absolue la chute d'Adam et le dépit du diable, mais constituait d'emblée le point d'arrivée de l'accomplissement du premier homme. L'histoire de la sainte économie est la théologie d'événements qui apportèrent leur contribution du début à la fin et qui, par conséquent, ne se laisse pas enfermer de façon univoque dans le modèle sotériologique « de la chute et du rachat », mais s'étend au contraire de la création aux confins du Royaume. C'est précisément pour cette raison que le salut dans le Christ n'est pas simplement un moyen de sortir du péché originel mais la réalisation du plan initial de l'union du créé et de l'incréé. Ce salut ne se réduit pas à une thérapie de la nature déchue mais contribue à la divinisation de la vie dans le Christ. Dans la perspective christique de l'homme, la chute et le péché, malgré tout le tragique qu'elles comportent, ne constituent pas l'épicentre de la création, pas davantage qu'un obstacle ontologique et éternel, mais un événement négatif, relatif et réversible qui n'est pas en opposition radicale avec le salut dans le Christ.

16 Jean Damascène, Exposé exact de la foi orthodoxe 77.

17 Augustin d'Hippone, Sermo 174, 2, PL 38, 940.

18 Athanase le Grand, De Inc. 54, PG 25, 192B.

19 Grégoire Palamas, The One Hundred and Fifty Chapters, 54, 146-148. 


\section{LA SYNERGIE DANS L'CUUVRE DE L'INCARNATION ET DU SALUT}

Le mystère de l'incarnation en tant qu'union du créé et de l'incréé dans l'hypostase du Verbe repose sur l'énergie et la grâce du Saint-Esprit accordée à la Vierge Marie. Les paramètres humain et divin contribuent de façon décisive, selon leur mode propre, à sa réalisation, de sorte que la vérité de l'incarnation dépende de l'intégrité et de la plénitude à la fois de la divinité et de l'homme. L'initiative de l'incarnation revenait incontestablement à la bienveillance du Dieu triadique. Mais si l'humanité ne participait pas, elle aussi, volontairement à l'incarnation du Fils, toute la procédure aurait été de ce fait imposée. Puisqu'il n'existe aucune relation essentielle entre Dieu incréé et l'homme créé, une réquisition forcée du créé, sans sa libre adhésion et son libre consentement, ne serait que désagrégation et anéantissement existentiel de la nature humaine. La grâce divine, " qui toujours guérit les maux et comble les carences $»,{ }^{20}$ ne vient pas peser de tout son poids comme un fardeau sur les épaules de l'homme. La bienveillance et I'initiative divines exigeaient comme condition incontournable le consentement libre et énergique de l'homme. La condition de cette libre procédure est remplie en la personne de la Vierge Marie et marque l'avènement de la divine incarnation, donnant naissance à la libre existence personnelle incarnée, qui relève du libre consentement de l'humanité. ${ }^{21}$ Le principe de cette synergie ${ }^{22}$ entre Dieu et l'homme vaut pour chaque homme pris séparément dans le mystère de son assimilation au corps du Christ. Le mystère de la grâce relève du libre consentement du croyant à l'œuvre du salut.

La vie dans le Christ, vécue avant tout à travers les mystères de l'Église et qui s'étend à la vie personnelle et sociale, constitue un don de la grâce. Toutefois, la bénédiction et l'accomplissement des croyants présupposent la liberté et la coopération de l'homme pour accueillir, préserver et faire fructifier cette grâce accordée par les mystères. La pratique chrétienne n'est pas une sorte de gymnastique intellectuelle mais la libre participation à la Passion du Christ en tant que dépouillement (kénose) par amour et rencontre avec l'autre dans un lien de fraternité.

20 Prière d'ordination de l'évêque, cf. L'Euchologio Barberini GR. 336, Edizione a cura di Stefano Parenti, ed. Elena Velkovska, Roma 1995, column 157.3, 174.

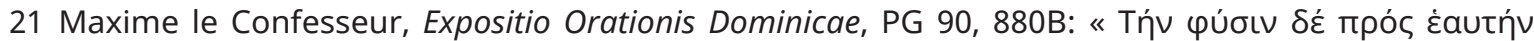

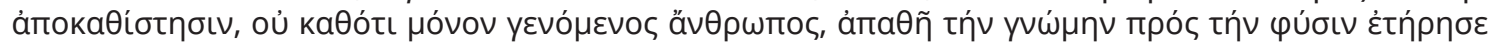

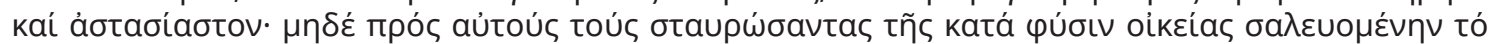

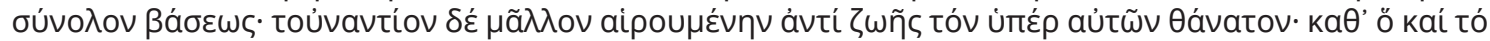

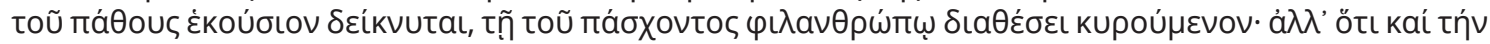

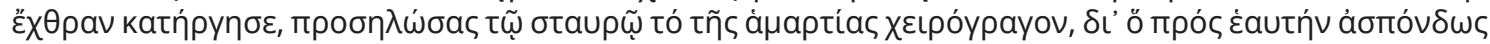

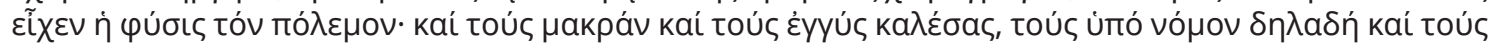

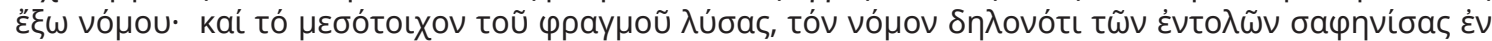

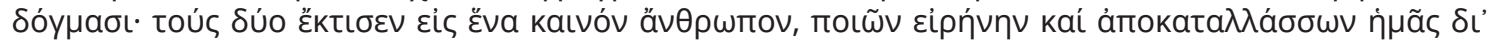

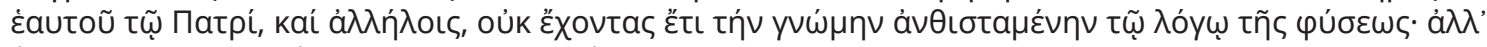

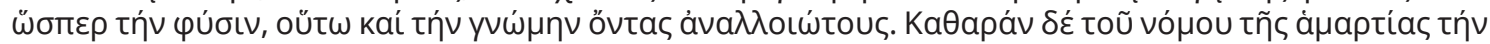

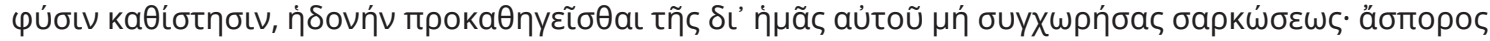

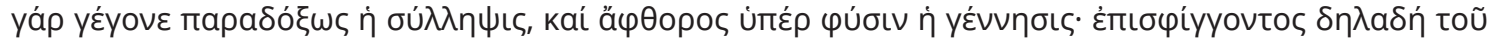

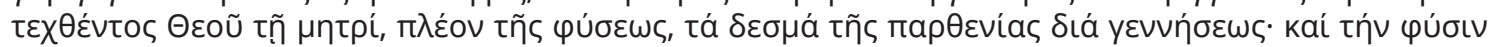

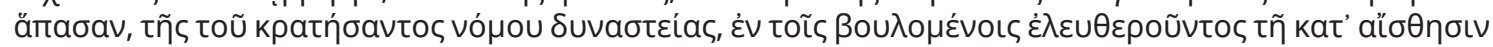

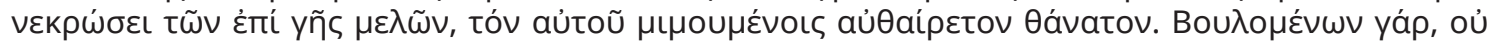

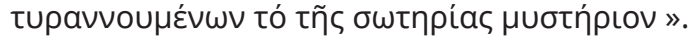

22 Cf. Jean Chrysostome, In Matthaeum Homil. 82, 4, PG 58, 742. In Epist. ad Ephes. 1, 2, PG 62, 12. In Inscriptionem Actorum, PG 51, 80. Maxime le Confesseur, Ambigua, PG 91, 1076AB. 
Il s'agit fondamentalement de l'intensité dialectique entre l'« ancien » et le « nouvel » homme, en tant que vie selon l'esprit et non selon la chair. Dans la tradition orthodoxe, la lutte contre les passions, la pratique de la vertu, la prière suivie, sont des réalités intimement liées à la participation aux mystères de l'Église. La bénédiction des dons et I'influence qu'exerce sur eux la grâce divine ne dépend pas de la pureté de l'homme pas plus que de la valeur du prêtre ou des croyants. "La grâce imprègne toute chose ${ }^{23}$. Le Christ fait appel aux saints dans le partage du pain de vie. Il ne s'agit véritablement pas d'une sainteté qui s'acquiert simplement d'une vertu souveraine, isolée de la vie des mystères. La sainteté constitue une participation eucharistique au Saint unique. L'Église dans son ensemble est qualifiée de sainte, de même que les croyants, selon la directive liturgique «Aux Saints les choses saintes », car ils participent au seul Saint qui soit et partagent son corps et son sang. En tant que partie du corps du Christ, les saints font affluer, à travers les mystères, la vie et la bénédiction à partir de la tête et du cœur de ce corps. Cette hypostase ecclésiologique de la synergie dans la vie dans le Christ signifie que le salut n'est pas une caution individuelle mais l'intégration à un tissu de relations et à la diversité des grâces et des liturgies dans le Corps du Christ. L'autorité et l'interprétation des Saintes Écritures, en tant qu'expérience partagée et témoignage au sein du Corps du Christ, est à mettre sur le même plan ecclésiologique. Dans le cas contraire, toute lecture personnelle, toute herméneutique subjective des Écritures apparaîtrait comme une implacable objectivité, comme une idéologie. Pourtant, sans un consentement libre et personnel, la bénédiction des croyants et la transmission de la grâce ne peuvent se réaliser. La grâce n'a pas de prise sur des êtres dépourvus de liberté et de volonté pas plus qu'elle n'est une forme quelconque de récompense. La grâce peut réaliser le salut, mais I'homme régénéré par Dieu, dont il est l'image, participe librement à ce mystère, mettant en accord sa volonté avec celle de Dieu, ayant comme objectif ultime « de partager la volonté de Dieu ». ${ }^{24}$

Combattant le pouvoir pontifical et son centralisme institutionnel, ses catégories métaphysiques abstraites et juridiques de la théologie scholastique, ainsi que sa conception religieuse inhumaine des mérites des œuvres, du purgatoire et du rachat des péchés, Luther assura la transition entre le Moyen Âge et la Renaissance. Se fondant sur les positions d'Augustin, Luther considérait que l'homme, en raison du péché originel, s'était totalement privé de Dieu à l'image de qui il avait été créé et était devenu une image du diable (imago diavoli). Déchu de la grâce divine, il fut puni d'avoir été coupable de la transgression dont il était l'auteur. Depuis lors, le péché personnel d'Adam est transmis à sa descendance. La corruption et la dépravation de l'homme est radicale et absolue (peccatum radicale). Amputé de la grâce, tout ce que l'homme entreprend se situe aux frontières du péché. Affirmant le total effacement en l'homme de l'image de Dieu, qu'il comprend statiquement comme s'identifiant à la ressemblance de Dieu, Luther n'entrevoit aucun mouvement dynamique ni aucune synergie de l'homme vers Dieu dont il a été créé à la ressemblance. L'homme ne peut plus désormais rien faire de lui-même pour son salut. ${ }^{25}$ Seule la grâce venant de l'Évangile et principalement du sacrifice de la croix du Christ peut réaliser la rédemption de I'homme. La foi comme acceptation de la volonté divine et la grâce sont les seuls auteurs du salut.

23 Nicolas Cabasilas, Explication de la Divine Liturgie, 47, 8-10.

24 Nicolas Cabasilas, La vie en Christ 7, 47.

25 Martin Luther, Explication du Psaume LI, trad. J.- F. Nardin, Toulouse (Cadaux) 1842, 13. 
Face à une conception qui considère comme intacte la liberté de I'homme (libre arbitre, liberum arbitrium) depuis la chute, Luther oppose une conception de la totale corruption et inexistence de la liberté (serf arbitre, servum arbitrium) ${ }^{26}$ prise en otage désormais par la nécessité du péché. La volonté de l'homme n'est nullement en mesure de se mettre librement en mouvement vers le salut. Le libre arbitre ne relève que de Dieu alors que les hommes sont aveuglément asservis au diable. La déchéance totale de l'image de Dieu et l'importance première accordée au péché va orienter la théologie protestante vers un enseignement déterministe de la seule prédestination. ${ }^{27}$ Pour Luther, la prévision et la prédestination sont une seule et même chose. Tout ce qui apparaît comme une action libre de I'homme, y compris la trahison de Judas, est en fin de compte prédestiné par la volonté de Dieu. «Puisque Dieu est en mesure de prédire, cela signifie que les événements se produisent par nécessité ». L'homme n'est de toute façon pas libre : d'une part, il est asservi à la nécessité de la toute-puissance, de la prévision, de la prédestination, de la volonté et de la grâce de Dieu et d'autre part, il est asservi au péché et à la mort. Seules la foi christocentrique et la grâce transcendante peuvent libérer I'homme du péché et soumettre sa volonté à la grâce divine comme « liberté royale » (regia libertas). La seule connaissance du péché n'est pas suffisante pour s'en acquitter. C'est pourquoi tous les commandements de l'Écriture ne sont rien d'autre que des commandements de Dieu adressés à l'homme pour lui indiquer ce qu'il doit faire et non ce qu'il peut faire. Ayant toujours à l'esprit les mérites des œuvres et la rédemption des péchés, Luther souligne que l'homme ne peut rien entreprendre qui relève de sa libre volonté et qui puisse avoir une quelconque influence dans son salut. Tout arrive par le Christ et le Saint-Esprit et l'homme ne joue qu'un rôle passif dans sa rédemption. ${ }^{28}$

Et même lorsqu'il est l'auteur d'actions bonnes, l'homme n'est pas lui-même l'auteur des œuvres de la foi, mais il est animé par la grâce et la foi en elles. La personne juste fait les œuvres, mais les œuvres ne justifient pas la personne. Pour Luther, I'homme d'après la chute est un organe ou un substrat de Dieu ou du diable..$^{29}$ L'homme se libère de la souveraineté du diable non grâce à ses forces propres, mais par la grâce de Dieu. L'homme simplement tombe sous le joug de la souveraineté de Dieu. ${ }^{30}$

Comme réveil du repentir et prise de conscience du péché, ce total abandon à la volonté de Dieu constitue la « vraie synergie »31 et non la « synergie hérétique » de I'homme dans l'œuvre du salut. « Dieu a soustrait mon salut de l'initiative de ma propre volonté et l'a confié à la sienne. Il me promet de me sauver, non en raison de mes actions et de mes efforts, mais en raison de sa grâce et de sa miséricorde... j'invite tous les hommes à lui prêter obéissance ».

26 Cf. Augustin d'Hippone, Contra Julianum II, 8, 23, PL 44, 689. Cf. Lopes, 2013. Vestrucci, 2019.

27 Martin Luther, « Du Serf Arbitre », Oeuvres V, Labor et Fides, Genève 1958, 149-151. Pour la relation de Luther avec l'Église orthodoxe, cf. Wendebourg, 1986. Podskalsky, 1988. Hering, 1968. Vischer, 1986. Pricop, 2013. Meimaris, 2013. Saarinen, 2014. Delikostantis, 2014. Asproulis, 2017.

28 Martin Luther, « Du Serf Arbitre »,124.

29 Martin Luther, « Du Serf Arbitre », 185.

30 Martin Luther, « Du Serf Arbitre », 190.

31 Le Père Dumitru Staniloae considère que tant Luther que la théologie protestante contemporaine (Barth, Stuhlmacher, Jüngel, Moltmann) peuvent détecter un rôle énergétique particulier de l'homme dans le processus de réalisation personnelle de son salut et de sa justification. Cf. Staniloae,1983. 
Toutes les étapes de la rédemption ou du salut de l'homme sont franchies par des voies transcendantes et par la grâce divine (ergo sola gratia justificat), sans la participation active de l'homme. La seule chose qui s'impose, c'est la foi par la grâce dans le Verbe et les actions de Dieu. Si la Loi exige des œuvres, l'Évangile exige la foi. Tout ce qui concerne le salut ne dépend et ne vient que de la grâce de Dieu et non de l'autorité des institutions ecclésiastiques, des actions ou de la volonté de l'homme. Et les œuvres de la foi sont elles aussi des dons de Dieu. Si la foi est reconnue par Dieu, le Saint-Esprit procède à ses œuvres. Le triptyque sola scriptura, sola fide, sola gratia, annule emblématiquement en fin de compte la libre volonté de l'homme. Et même le Baptême de l'Église n'efface pas le péché, profondément ancré en l'homme, mais simplement le pardonne et n'incrimine pas son auteur. L'homme obtient la rémission de ses péchés car il est désormais sous le joug du Christ qui l'a tenu captif dans son Esprit de sorte qu'il ne veut et ne fait de bon gré que ce que veut le Christ. Cet « heureux changement », celui de la divinisation de I'homme ${ }^{32}$ est vécu dialectiquement plutôt comme un espoir et comme une promesse ; sa plénitude, en raison de la survivance du péché en l'homme ne se réalisera qu'aux fins dernières. Ainsi la nature humaine est libérée, mais n'est pas pour autant guérie de la culpabilité universelle devant le péché. C'est pourquoi l'homme, dans la phase actuelle de son évolution, est paradoxalement « en même temps juste et pêcheur » (simul justus et peccator) ${ }^{33}$. Le péché est si profondément incrusté dans la vie de l'homme que son total effacement et la plénitude du salut ne peuvent qu'être renvoyés à la vie future.

Cette situation offre un terrain propice à l'autonomisation ultérieure de la réalité naturelle et matérielle par rapport au niveau spirituel et métaphysique. Ce fait explique sans doute pourquoi la sotériologie de Luther est de façon univoque anthropomoniste, le monde comme initiative et création de Dieu y étant absent.

L'existence endocosmique de l'homme se présente comme étant totalement coupée de l'œuvre de la rédemption par la foi et la grâce de Dieu. Ainsi se fait jour le paradoxe selon lequel la volonté de I'homme, soumise à Dieu ou au diable, d'atteindre les réalités supérieures transcendantes du salut, est libre et autonome uniquement lorsqu'elle s'exerce par rapport à ce monde, lorsqu'elle intervient sur des questions de survie, dans l'action au niveau social, économique, scientifique et politique. Il s'agit en quelque sorte d'une espèce de «liberté de nature ", liée à la faculté noétique de I'homme et à la faculté de se déterminer volontairement pour la régulation des affaires de ce monde et la pratique de bonnes conduites naturelles à l'égard des hommes (coram hominibus).

32 Il est caractéristique que le dialogue orthodoxe-luthérien au cours des quatre dernières décennies ait créé les conditions pour l'acceptation par les luthériens du terme théosis et son association avec les termes les plus familiers à la sotériologie de Luther comme justification et sanctification. En effet, le luthéranisme finlandais dans son dialogue avec les théologiens orthodoxes a particulièrement développé cette théologie herméneutique chez Luther, parlant du partage de la vie divine de manière réaliste et ontologique. Cf. Saarinen, 2014. Mannermaa, 1989. Heubach1990. Marquart, 2000. Cependant, cette ouverture œcuménique est critiquée par une grammaire herméneutique luthérienne plus « orthodoxe », qui renvoie à différentes conditions ontologiques et interprétatives de la sotériologie de Luther à l'égard de l'enseignement orthodoxe des Pères grecs de l'Église. Voir par exemple, Ebeling, 1990. Flogaus, 1996. Flogaus, 1997. Flogaus, 1999.

33 Selon Nikos Nisiotis, "l'être humain est une image de Dieu et en même temps un pécheur » est la réalité la plus évidente du monde moderne. Cependant, ces deux dimensions doivent être maintenues en tension constante, sachant que cette dualité peut être surpassée de manière eschatologique par l'espérance, le repentir et la volonté de liberté en faveur de l'image de Dieu, qui le libre arbitre vainc le péché. Cf. Nissiotis, 1981. 
Cette volonté n'est nullement en état de se mesurer à Dieu pour sa rédemption et son salut (Justitia coram Deo) ${ }^{34}$.

Luther qualifia ce paradoxe de monoénergisme et de monothélisme, tentant ainsi d'interpréter la conception paulienne de la rédemption à travers la foi et non à travers les œuvres de la Loi (Romains 1,17 ; 3, 21-30). Sa position se situe dans un cadre historique précis, marqué par les particularités de son époque. Face à une piété de masse reposant sur des superstitions de toutes sortes, sur les mérites et le rachat financier des péchés, Luther oppose une vie existentielle et personnelle de la foi, nourrie et augmentée par la Bible (sola scriptura), alors que dans le même temps il désacralise la médiation horizontale du pouvoir ecclésiastique et politique. L'enseignement de Luther ne provoqua pas seulement un violent conflit religieux, qui divisa l'Europe chrétienne, mais conduisit également plus largement à une évolution révolutionnaire de la civilisation de la modernité qui avait été amorcée à la Renaissance. Il déplaça la foi obligatoire et impersonnelle, qui se cachait derrière les bonnes œuvres d'un christianisme de masse mettant l'accent sur les dons coutumiers, les actes de vénération, les reliques, les offrandes matérielles pour le rachat des péchés, vers d'autres horizons, ceux du vécu personnel et responsable de la foi comme don de la grâce de Dieu. Il souligne le caractère profondément existentiel et ontologique tant du péché que de la foi, au-delà de l'optimisme de l'héritage du donatisme et du pélagianisme dans la tradition chrétienne occidentale.

Cette individualisation religieuse pourrait éventuellement réduire le caractère ecclésiologique de la foi et conduire à un confessionnalisme sectaire et à la division. Le retrait de la volonté libre de l'homme de l'œuvre du salut fit ressortir le caractère charismatique et transcendant de la foi et de la grâce, au-delà de médiations hiérocratiques et des mérites. L'homme, bien qu'il n'ait pas un libre arbitre, devient à la fois esclave et libre par la vie chrétienne, la foi et la parole de l'Évangile ${ }^{35}$. Pourtant, non seulement il a élaboré une conception de la liberté naturelle indépendante de la foi, mais il a aussi libéré les forces volitives et créatrices de la liberté humaine et les a orientées vers une conception sécularisée de la rédemption à travers les œuvres endocosmiques. L'histoire, mais également l'Église visible et ses institutions hiérarchiques, ou encore les œuvres des hommes, ne pouvaient être le lieu du salut. Luther lui-même exprima personnellement et empiriquement cette position, en opposition avec l'ordre ecclésiastique et religieux établi de son époque.

Sa position et son enseignement libérateurs au sujet de la rédemption à travers la foi provoqua une révolution sur le plan religieux dont les conséquences sociopolitiques réelles constituèrent la matrice de toute révolution ou réforme en politique. La pensée critique, les Lumières, la liberté de culte, les droits de l'homme, la séparation de l'Église et de l'État, l'existentialisme, n'auraient jamais pu voir le jour dans I'histoire récente de l'Europe, sans l'apport et l'influence du protestantisme. L'exemple personnel et l'enseignement de Luther sur la question de la rédemption, tel qu'il a été reçu et tel qu'il s'est développé ultérieurement, constitua la matrice qui donna naissance au sujet rationnel moderne. Dans la vie et les œuvres des hommes, il n'y a rien d'immuablement sacré.

34 Cf. Martin Luther, « Du Serf Arbitre », 7-236.

35 Tel est le contenu du célèbre ouvrage de Luther pour la liberté chrétienne, voir Martin Luther, Le Traité de la Liberté Chrétienne, Fuvres I, Labor et Fidès, Genève 1966, 275-306. 
Proclamant la nécessité d'une permanente réforme de l'Église, cette idée semble avoir joué un rôle décisif dans le progrès et l'évolution de l'histoire moderne. Avec le protestantisme, la voie du modèle de civilisation occidentale semble avoir déplacé l'activité de I'homme du champ de la foi métaphysique vers une réalité endocosmique émancipée ${ }^{36}$. La civilisation de la modernité dans son ensemble et ses prolongements sont liés en quelque sorte à l'action de l'homme libérée de toute autorité métaphysique, dans le champ de la science, de la technologie et de la culture.

Malgré la relation qu'entretient la modernité avec l'évolution de la tradition chrétienne en Europe, il est temps, à l'époque que nous vivons, de faire observer que les Églises chrétiennes ne sont plus désormais le moteur actif de l'histoire, mais au contraire le spectateur passif et sans influence des bouleversements et des événements. Les réformes sociales et économiques, la libération et l'émancipation des peuples et des classes sociales, les effets désastreux du nationalisme et du totalitarisme, le progrès technologique, le nihilisme consumériste et la destruction catastrophique de l'environnement naturel, le nouvel ordre des choses, les formes postmodernes de "spiritualité » ainsi que toute tentative d'évasion des difficultés de la vie etc. sont l'œuvre de facteurs du monde présent, coupés de la foi chrétienne, voire en opposition avec elle.

Si Luther vivait à notre époque, ses priorités sur le plan théologique seraient sans aucun doute très différentes. En raison de la grâce, elles constitueraient peut-être une initiative alternative de réconciliation des chrétiens divisés et un témoignage public concret du salut par le Christ dans le monde d'aujourd'hui radicalement transfiguré, dans lequel, au-delà du péché individuel, règne à un échelon désormais collectif et généralisé le mal sous toutes ses formes, l'aliénation, le vide, le malaise de la civilisation et l'absence de sens.

\section{BIBLIOGRAPHIE}

Nikolaos Asproulis (2017): «The Encounter between Eastern Orthodoxy and Lutheranism, A Historical and Theological Assessment », The Ecumenical Review 69.2/2017, pp. 215-224.

Jean Daniélou (1944) : Platonisme et théologie mystique, doctrine spirituelle de saint Grégoire de Nysse, Paris ${ }^{2} 1944$.

Konstantinos Delikostantis (2014): «Orthodox Dialogues with the Lutheran Churches », Orthodox Handbook on Ecumenism. Resources for Theological Education, edid. by P. Kalaitzidis, T. Fitzgerald, C. Hovorun, Volos-Geneva-Oxford, 2014, pp. 473-477.

Gerhard Ebeling (1990): «Der Sühnetod Christi als Glaubensaussage: Eine hermeneutische Rechenschaft », Zeitschrift für Theologie und Kirche, (Die Heilsbedeutung des Kreuzes für Glaube und Hoffnung des Christen) Beiheft 8/1990, pp. 3-28.

Gerhard Ebeling (2012) : «Thèses sur la doctrine des deux règnes » (1972), in Pierre Bühler (edid.), Répondre de la foi. Réflexions et dialogues, Textes de Gerhard Ebeling, Labor et Fides, Genève 2012, pp. 139-158.

Reinhard Flogaus (1996): « Einig in Sachen Theosis und Synergie? », Kerygma und Dogma 42/1996, pp. 225-243.

Reinhard Flogaus (1997): Theosis bei Palamas und Luther. Ein Beitrag zum ökumenischen Gespräch, Vandenhoeck \& Ruprecht, Göttingen 1997.

36 Cf. Ebeling, 2012, 139-158. Lienhard, 1976, 25-41. Thompson, 1984. Laffin, 2016. 
Reinhard Flogaus (1999): «Agreement on the Issues of Deification and Synergy? », Luther Digest. An Annual Abridgement of Luther Studies 7/1999, pp. 99-105.

Gunnar Hering (1968): Ökumenisches Patriarchat und europäische Politik 1620-1638, Wiesbaden 1968.

Joachim Heubach (1990): Luther und Theosis, Veroffentlichungen der Luther-Akademie Ratzeburg, Band 16, Martin-Luther Verlag, Erlangen 1990.

Michael Laffin (2016): The Promise of Martin Luther's Political Theology, Freeing Luther from the Modern Political Narrative, Bloomsbury T\&T Clark, London/New York 2016.

Marc Lienhard (1976) : « La doctrine des deux règnes et son impact dans I'histoire », Positions lutheriennes 24/1976, pp. 25-41.

Pereira Jairzinho Lopes (2013): Augustine of Hippo and Martin Luther on Original Sin and Justification of the Sinner, Vandenhoeck \& Ruprecht, Gottingen 2013.

Tuomo Mannermaa (1989): Der Im Glauben Gegenwdrtige Christus: Rechtfetigung und Vergottung. Zum iikumenischen Dialog. Arbeiten zur Geschichte und Theologie des Luthertums, Neue Folge, Band 8 Lutherisches Verlagshaus, Hannover 1989.

Kurt E. Marquart (2000): «Luther and Theosis », Concordia Theological Quarterly 64.3/2000, pp. 182-205.

Theodore Meimaris (2013): «Thirty Years of the International Theological Dialogue between Orthodox and Lutherans (1981-2011): Evaluation and Prospects», Nicolaus 40.1/2013), бб. 159-186.

Panayotis Nellas (1987): Deification in Christ. The Nature of the Human Person, trans. N. Russel, St. Vladimir's Seminary Press: Crestwood, NY 1987.

Nikos Nissiotis (1981) : « L'homme image de Dieu et pecheur, L'umanisme contemporain et la théologie de la liberation ", Annuaire académique de la Faculté de théologie de l'Université d'Athènes 25/1981, 331-360.

Gerhard Podskalsky (1988): Griechische Theologie in der Zeit der Türkenherrschaft (1453-1821), Die Orthodoxie im Spannungsfeld der nachreformatorischen Konfessionen des Weltens, München 1988.

Cosmin Pricop (2013): From Espoo to Paphos: The Theological Dialogue of the Orthodox Churches with the Lutheran World Federation (1981-2008), Bucharest 2013.

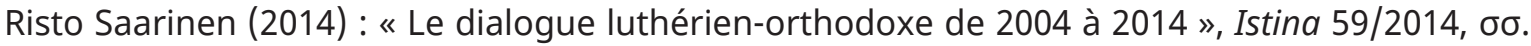
367-386.

Dumitru Staniloae (1983) : « Le sens de la justification chez Luther. Quelques remarques orthodoxes ", Luther et la Réforme allemande dans une perspective oecuménique, Études théologiques de Chambésy 3, Centre Orthodoxe, Chambésy-Genève 1983, pp. 185-195.

Cargill Thompson (1984): The political Thought of Martin Luther, Philip Broadhead, edited by Philip Broadhead and with a preface by A.G. Dickens, The Harvester Press-Barnes \& Noble Books, Brighton-Totowa 1984.

Andrea Vestrucci (2019): Theology as Freedom. On Martin Luther's 'De servo arbitrio', Mohr Siebeck, Tübingen 2019.

Lukas Vischer (1986): «The Legacy of Kyrill Loukaris: A Contribution to the Orthodox-Reformed Dialogue », Mid-Stream 25.2/1986, бб. 165-183.

Dorothea Wendebourg (1986): Reformation und Orthodoxie. Der ökumenische Briefwechsel zwischen der Leitung der Württembergischen Kirche und Patriarch Jeremias II. von Konstantinopel in den Jahren 1573-1581, Göttingen 1986.

Stavros Yangazoglou (2001): Communion of Theosis, The synthesis of Christology and Pneumatology in the work of Gregory Palamas, Athens 2001 (in Greek).

Jean Zizioulas (1984) : “Christologie et existence. La dialectique créé-incréé et le dogme de Chalcédoine", Contacts 36/1984, pp. 153-173. 


\section{SIN, FREEDOM AND FREE WILL}

\section{Summary:}

According to the theology of the Greek Fathers of the Church, the image of God in man is mainly located in the free will of nature, in his/her ability to exist as a free person. In other words, man desires to exist because he/she loves, accepting the loving call of God.

Man freely rejected and abandoned the vivifying grace, which was its ultimate point of reference and the core of its existence, putting an end to the divine plan. It is not simply a question of negative psychological behavior on the ethical level, but primarily of an ontological choice. Man realizes his life in a self-sufficient and autonomous way, renouncing its relationship and communion with God.

Man, the only being in the created world, among beings endowed with or without reason, had the privilege of uniting the created with the uncreated. The image of God and the free will of man continue to exist after the fall and sin. Therefore, it is through man that creation had to be reconstituted. The mystery of the incarnation as a union of the created and the uncreated in the hypostasis of the Word takes place through the energy and grace of the Holy Spirit on the Virgin Mary. The human and divine factors contribute decisively, according to their own mode, to its realization, so that the truth of the incarnation depends on the integrity and the fullness of both the God and man.

Based on Augustine, Luther considered that man, because of the original sin, had been totally deprived of the image of God and had become an image of the devil. Fallen from divine grace, he was punished for having been guilty of the transgression of which he was the author. The corruption and depravity of man is radical and absolute. Bereft of grace, all that humanity undertakes is on the frontiers of sin. Affirming man's full obliteration of God's image, which he statistically understands as being identified with the likeness of God, Luther does not see any dynamic movement or synergy of man towards the likeness. Man can no longer do anything by himself for his salvation. Only grace from the Gospel and mainly the sacrifice of the Cross of Christ can realize the redemption of man. Faith alone as acceptance of the divine will and grace are the only authors of salvation.

Man, although although he does not have free will, becomes simultaneously slave and free by the Christian life, faith and the word of the Gospel. This religious individualization develops a conception of natural freedom independent of faith and liberated the volitional and creative forces of human freedom and directed them to a secularized conception of redemption through immanent works. History, but also the visible Church and its hierarchical institutions, or the works of man, could not be considered as the place of salvation. Luther himself personally and empirically expressed this position, in opposition to the established ecclesiastical and religious order of his time. His liberating position and teaching about redemption through faith provoked a religious revolution whose real socio-political consequences constituted the source of any revolution or reform in politics. Luther's personal example and teaching on the question of redemption, as it was received and as it later developed, was the source that gave birth to the modern rational subject. In the life and works of humanity, there is nothing immutably sacred. Proclaiming the need for a permanent reform of the Church, this idea seems to have played a decisive role in the progress and evolution of modern history.

With Protestantism, the Western model of civilization seems to have displaced man's activity from the field of metaphysical faith to an emancipated immanent reality. The culture of modernity and its extensions are in some way linked to the action of man freed from all metaphysical authority, in the field of science, technology and culture.

\section{Keywords:}

Patristic tradition, Reform, Luther, anthropology, hermeneutics, creation, image and likeness, free will, serf referee, peach, freedom, deification, synergy, $\mathrm{Hi}$, modernity, redemption, justification. 